\title{
An L-lactate Biosensor Based on Printed Organic Inverter Circuitry and with a Tunable Detection Limit
}

\author{
Kuniaki Nagamine, ${ }^{1 *}$ Taisei Mano, ${ }^{1}$ Rei Shiwaku, ${ }^{1}$ Hiroyuki Furusawa, ${ }^{2}$ \\ Hiroyuki Matsui, ${ }^{1}$ Daisuke Kumaki, ${ }^{1}$ and Shizuo Tokito ${ }^{1 * *}$ \\ ${ }^{1}$ Research Center for Organic Electronics (ROEL), Yamagata University, \\ 4-3-16 Jonan, Yonezawa, Yamagata 992-8510, Japan \\ ${ }^{2}$ Graduate School of Science and Engineering, Yamagata University, \\ 4-3-16, Jonan, Yonezawa, Yamagata 992-8510, Japan
}

(Received October 31, 2018; accepted December 13, 2018)

Keywords: printed electronics, organic inverter circuit, biosensor, limit detection, wearable

In this study, we showed proof-of-principle experiments to demonstrate a printed organic inverter-circuit-based enzyme sensor with an extended-gate electrode with a lactate oxidase (LOx) and Prussian Blue carbon-graphite ink active area for the first time to inform users of an L-lactate overlimit concentration via a simple integrated electrophoretic display. The twostage organic inverter circuit exhibited an abrupt shift in output voltage depending on the potentiometric sensor signal imposed on the constant input voltage of the inverter circuit, resulting in a distinct color change of the display connected to the output line of the inverter circuit. The L-lactate concentration limit was tuned by changing the input voltage of the inverter circuit. This new type of wearable biosensor, notifying the user of an overlimit condition, can arbitrarily be tuned by professionals such as medical doctors or sports trainers and would be helpful for users who do not have sufficient knowledge about the detected biomarkers.

\section{Introduction}

The recent development of wearable chemical sensor devices has enabled the daily monitoring of biomarkers from external bodily fluids such as tears, saliva, urine, and sweat. ${ }^{(1-6)}$ However, it is difficult for people with insufficient knowledge about biomarkers to understand their physical conditions from the detected signal. This is one of the issues to be addressed in realizing effective personal healthcare. Wearable sensors that can inform the users of only the overlimit signal arbitrarily tuned by a professional such as a medical doctor or a trainer could be beneficial for users in understanding their physical conditions. ${ }^{(7-9)}$ These sensors require a display that can convert the overlimit signal into a user-readable form. Some wearable sensors have been combined with a user-readable display such as an electrochromic ${ }^{(10-13)}$ or LEDbased display, ${ }^{(14,15)}$ which exhibits subtle color changes depending on the analyte concentration. It is crucial for a display to show a distinct signal change at the concentration limit of the

\footnotetext{
*Corresponding author: e-mail: nagamine@yz.yamagata-u.ac.jp

**Corresponding author: e-mail: tokito@yz.yamagata-u.ac.jp

https://doi.org/10.18494/SAM.2019.2174
} 
analyte. An inverter circuit is a logical gate that outputs reversed logic "zero" or logic "1" against the input of logic "1" (equivalent to the supply voltage $V_{D D}$ ) or logic "0" (equivalent to $0 \mathrm{~V}$ ), respectively. ${ }^{(16-19)}$ We considered that, if the input voltage of the inverter circuit can be controlled by the potentiometric output of the enzyme-based biosensor depending on the analyte concentration, the inverter circuit will exhibit steep switching of the output voltage at the threshold concentration of the analyte to induce a clear change in display signage. Recently, we have developed a printed organic transistor-based inverter circuit with low operating voltage. ${ }^{(20,21)}$ The printing technology has emerged as a low-cost, environment-friendly mass manufacturing technology for the fabrication of next-generation printed wearable flexible devices based on thin-film transistors. ${ }^{(22-27)}$

In the present study, we developed a unique organic inverter-circuit-based enzyme biosensor with tunable limit detection. The biosensing cell was composed of a carbon-based extendedgate electrode that is modified by an enzyme and the reference electrode was connected to the input line of the inverter circuit, and the output line of the circuit was connected to a simple electrophoretic display that shows a distinct color change. An L-lactate biosensing system was fabricated to demonstrate the operating mechanisms of the sensor device.

\section{Experimental Procedure}

\subsection{Device fabrication}

The fabrication process for the sensor device was similar to that in our previous report. ${ }^{(28)}$ In short, silver nanoparticle ink in a hydrocarbon-based solution (Harima Chemicals, NPS-JL) was printed on a glass slide or a polyethylene naphthalate (PEN) film substrate (125 $\mu \mathrm{m}$ thickness) as a gate electrode using an inkjet printer (Fujifilm, Dimatix DMP2831). After sintering silver nanoparticles for $30 \mathrm{~min}$ at $120{ }^{\circ} \mathrm{C}$ in air ambient, a 150-nm-thick parylene (KISCO, diX-SR) gate dielectric layer was formed by chemical vapor deposition. Then, the silver nanoparticle ink-based source and drain electrodes were subsequently printed. A bank layer (200 nm thick) composed of fluoropolymer ( $1 \mathrm{wt} \%$, DuPont, Teflon AF1600) in fluorinert (3M, FC-43) was then printed using a dispenser system (MUSASHI Engineering, Image Master 350 PC). After the treatment of the device with $30 \mathrm{mM}$ pentafluorobenzenethiol (Tokyo Chemical Industry) dissolved in 2-propanol for $5 \mathrm{~min}$ at room temperature, a toluene-based organic semiconductor (OSC) mixture containing $0.9 \mathrm{wt} \% p$-type OSC DTBDT-C 6 [Fig. A1(a), Tosoh Corporation] and polystyrene $(0.3 \mathrm{wt} \% \text {, Sigma-Aldrich, } M W \approx 280,000)^{(20,21)}$ was printed onto the area defined by the bank layer using the dispenser system. The resulting channel width and length were 780 and $18 \mu \mathrm{m}$, respectively. Finally, a parylene encapsulation layer ( $250 \mathrm{~nm}$ thick) was formed by chemical vapor deposition. The electrical characteristics of the fabricated organic transistor were evaluated immediately before use as shown in Supporting Figs. A1(b) and A1(c). The gate voltage $\left(V_{G}\right)$ of the device was swept from 5 to $-6 \mathrm{~V}$ while keeping the source-drain voltage $\left(V_{S D}\right)$ at $-2 \mathrm{~V}$ using the source meter (Keithley). All measurements were carried out in air ambient. 


\subsection{Fabrication of the enzyme/PB-modified extended-gate electrode}

The fabrication process for the electrode was similar to our previous report. ${ }^{(28)}$ In summary, a $\mathrm{Au}$ electrode pattern composed of an active area $\left(3 \times 5 \mathrm{~mm}^{2}\right)$ connected to a narrow lead line pattern ( $1 \mathrm{~mm}$ width, $3 \mathrm{~cm}$ length) was fabricated by thermal evaporation on a PEN film substrate (125 $\mu \mathrm{m}$ thickness) through a stencil mask. The active area was modified with carbongraphite ink including a redox compound of Prussian blue (PB-carbon) (C2070424P2, Gwent Inc.) and $10 \mu \mathrm{L}$ of a mixture solution composed of $1 \mathrm{U}_{\mu} \mathrm{L}^{-1}$ lactate oxidase (LOx, TOYOBO Co., Ltd.) in $100 \mathrm{mM}$ phosphate buffer (pH 6.8, Nakalai Tesque) and $0.1 \mathrm{wt} \%$ chitosan (Junsei Chemical) in $50 \mathrm{mM} \mathrm{HCl}(\mathrm{pH} \mathrm{5.4)}$ at the ratio $1.4: 10$. The resulting electrode was washed in stirred Dulbecco's phosphate buffered saline (DPBS) solution ( $\mathrm{pH} 7.4$, Sigma Aldrich) for $1 \mathrm{~h}$ to remove the LOx loosely bound to the chitosan gel.

\subsection{Potentiometric measurement using the extended-gate electrode with LOx/PB active area}

The LOx/PB-modified extended-gate electrode and the $\mathrm{Ag} / \mathrm{AgCl}$ reference electrode were immersed in the DPBS solution. The potential difference between the electrodes was monitored using an electrochemical analyzer (ALS model 602E, BAS Inc.) operated in an open-circuit potential mode (input impedance: $1 \times 10^{12} \Omega$ ). During the potentiometric measurement, the concentrated L-lactate dissolved in the DPBS solution was injected into the measurement solution and stirred at $350 \mathrm{rpm}$ to induce an enzymatic reaction at the active area of the extended-gate electrode. The temperature of the measurement solution was maintained at $30{ }^{\circ} \mathrm{C}$ using a hot plate.

\subsection{Detecting threshold concentration of L-lactate using the extended-gate electrode connected to the organic inverter circuit with a display unit}

The LOx/PB-modified extended-gate and $\mathrm{Ag} / \mathrm{AgCl}$ reference electrodes were immersed in the DPBS solution, and their terminals were connected to the gate and source electrodes of the first inverter, respectively. An electrophoretic display unit purchased from E Ink Corporation (SC001221, operating voltages of 5-15 V) was connected to the output line of the second inverter in parallel to the external resistor $2\left(R_{E X 2}\right)$. Real-time monitoring of $V_{O U T 2}$ with successive injections of a concentrated L-lactate solution into the DPBS solution was performed while applying constant voltages of $V_{I N 1}(5.35 \mathrm{~V})$ and $V_{D D}(6 \mathrm{~V})$.

\section{Results and Discussion}

Figure 1 shows a photograph of the printed biosensor device with the display unit [Fig. 1(a)] and its configuration [Fig. 1(b)]. The device is composed of three basic components: the biosensing cell, which consists of the extended-gate electrode modified with LOx/PB and the $\mathrm{Ag} / \mathrm{AgCl}$ reference electrode [Fig. 1(c)], a two-stage organic inverter circuit employing 
(a)

(b)

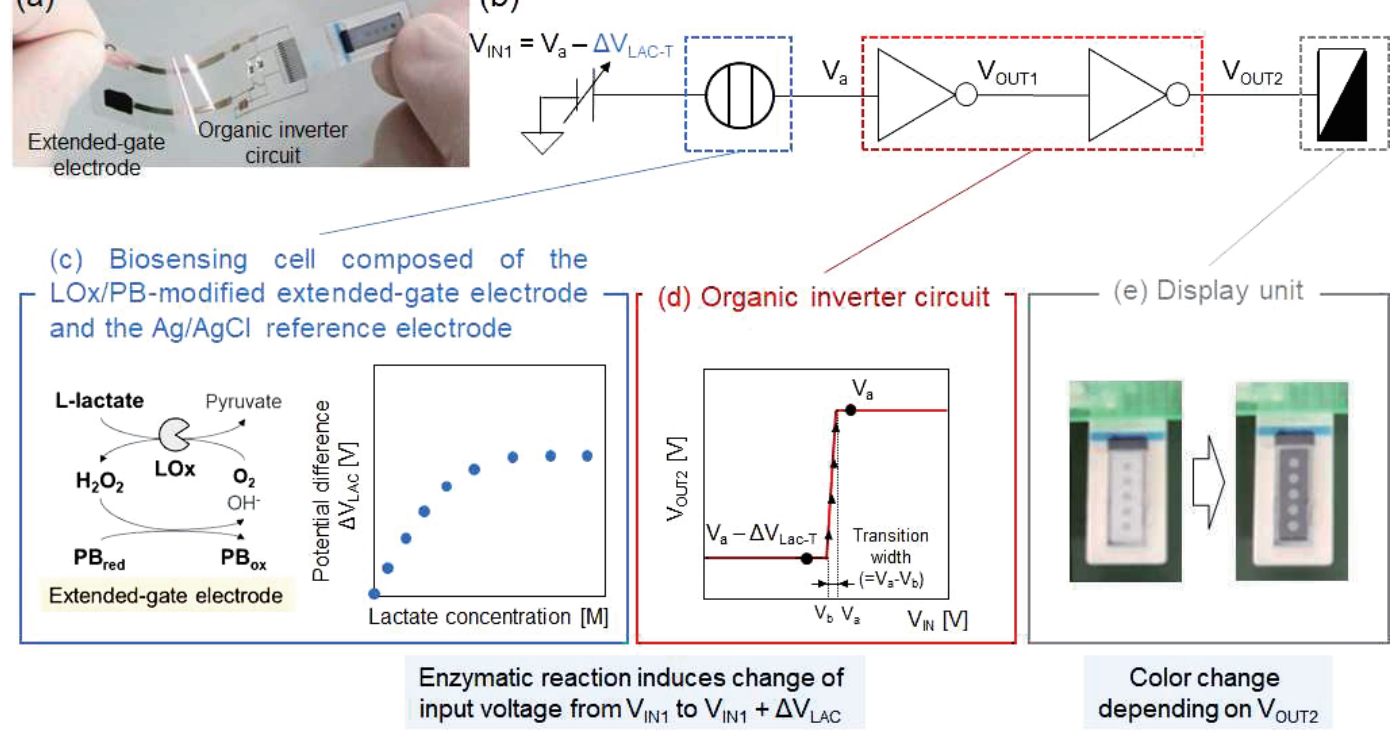

Fig. 1. (Color online) (a) Photograph of the printed organic inverter-circuit-based biosensor prototype device with the display unit and (b) its configuration, composed of three components, namely, (c) a biosensing cell composed of the extended-gate and $\mathrm{Ag} / \mathrm{AgCl}$ reference electrodes, (d) a two-stage organic transistor-based inverter circuit with a $p$-type semiconductor, and (e) a display unit.

a $p$-type semiconductor [Fig. 1(d)], and the display unit [Fig. 1(e)]. The extended-gate and reference electrodes were connected to the gate and source electrodes of the first inverter, respectively. In this configuration, the input voltage $V_{I N}$ was applied to the gate electrode of the first inverter, creating an electrical potential at the biosensing cell. L-lactate was oxidized by LOx while generating hydrogen peroxide, followed by the irreversible oxidation of PB from divalent to trivalent with hydrogen peroxide [Fig. 1(c)]. ${ }^{(29,30)}$ As a result, the electric potential of the extended-gate electrode changes $\left(\Delta V_{L A C}\right)$ according to the Nernst equation. $\triangle V_{L A C-T}$ for the concentration limit of L-lactate was calculated from the titration curve of the electrode with LOx/PB. The two-stage inverter was designed to exhibit a steep change in the output voltage $V_{\text {OUT2 }}$ with the same polarity as $V_{I N}$ within the narrow transition width $V_{a}-V_{b}$ as shown in Fig. 1(d). If the input voltage applied to the inverter via the biosensing cell was set to $V_{I N 1}=V_{a}-\Delta V_{L A C-T}\left(\Delta V_{L A C-T}\right.$ should be more than the transition width), the output voltage of the inverter $V_{O U T 2}$ steeply increased from $0 \mathrm{~V}$ to $V_{D D}$ after the injection of L-lactate overlimit concentration [Fig. 1(d)] followed by the color change of the display unit from white to black [Fig. 1(e)]. In this study, an electrophoretic display was used as an indicator because it is bistable and operates at relatively low voltages. Arbitrarily setting $V_{I N 1}$ depending on $\triangle V_{L A C-T}$ enables the tuning of the L-lactate concentration limit detected by the sensor device.

Figures 2(a) and 2(b) show the photograph (a) and circuit diagram (b) of the two-stage organic inverter circuit. The transfer and output curves of the single organic transistor are shown in Figs. A1(b) and A1(c). The field-effect mobility of the OSC in the saturation region was estimated to be $0.44 \mathrm{~cm}^{2} / \mathrm{Vs}$ and the on/off current ratio was $\sim 10^{8}$. The resistivities of the external resistors 1 and 2 [referred to as $R_{E X 1}$ and $R_{E X 2}$ in Fig. 2(b), respectively] were 


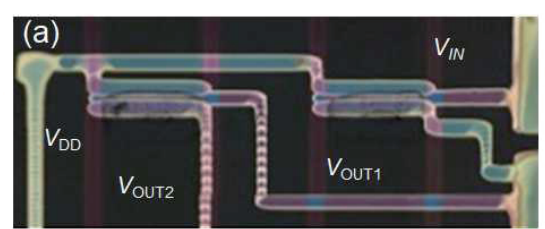

(b)

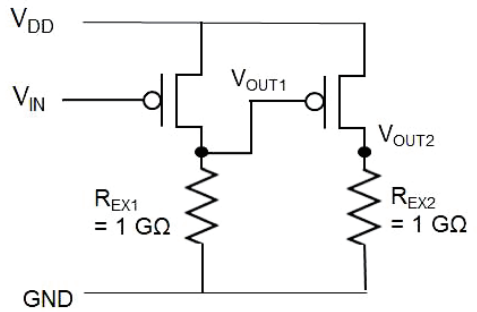

(c)

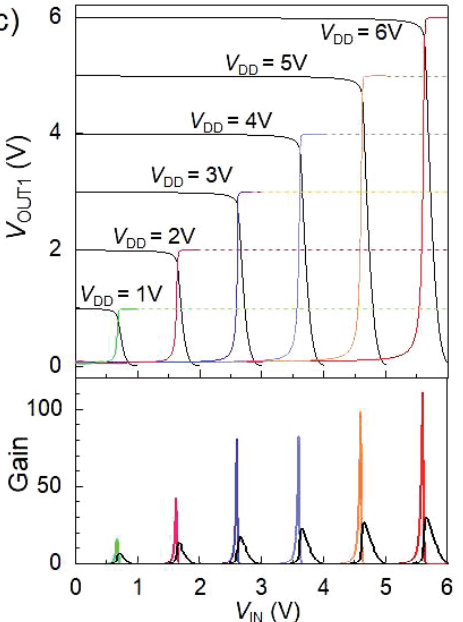

(d)

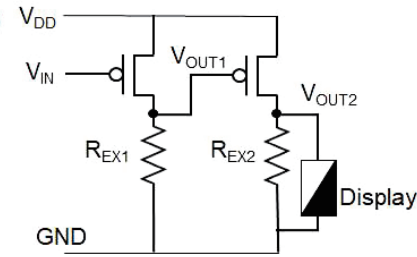

(e)

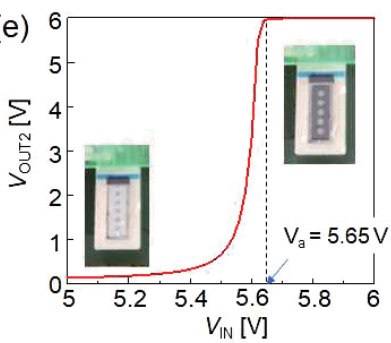

Fig. 2. (Color online) (a) Photograph and (b) circuit diagram of the two-stage organic inverter circuit. (c) Output voltages $\left(\mathrm{V}_{O U T 1}\right.$ and $\left.V_{O U T 2}\right)$ and gain as functions of input voltage $\left(V_{I N}\right)$. (d and e) Circuit diagram of the two-stage organic inverter circuit, where, as in the text, the electrophoretic display was inserted in parallel to $R_{E X 2}$ (c), and its output voltage $V_{\text {OUT2 }}$ as a function of $V_{I N}(\mathrm{e})$.

determined from the transfer characteristics of the device. Figure 2(c) shows the output voltages ( $V_{\text {OUT1 }}$ and $V_{\text {OUT2 }}$ for the first and second stages of inverters, respectively) and gain as functions of input voltage $\left(V_{I N}\right)$. $V_{O U T 1}$ switches from a value near $V_{D D}$ to zero at the first stage of the inverter during the linear increase in $V_{I N}$ from zero to the same value as $V_{D D}$. As a result, $V_{O U T 2}$ exhibited steeper switching from zero to a value near $V_{D D}$ owing to the sharp change in $V_{O U T 1}$ applied to the gate electrode of the second stage of the inverter. These characteristics of the two-stage inverter circuit were confirmed quantitatively from the gain curve derived from the $\left|\partial V_{O U T} / \partial V_{I N}\right|$ of the output voltage curve. In the following study, $V_{D D}$ was set to $6 \mathrm{~V}$ to operate the display that showed distinct color switching at more than 6 V. Figures 2(d) and 2(e) show the circuit diagram [Fig. 2(d)] of the two-stage organic inverter circuit, where the display was inserted in parallel to $R_{E X 2}$, and its output voltage $V_{O U T 2}$ as a function of $V_{I N}$ [Fig. 2(e)]. When $V_{I N}$ was increased to more than about $5.45 \mathrm{~V}, V_{\text {OUT2 }}$ increased sharply, and $V_{O U T 2}=6 \mathrm{~V}$ at $V_{I N}=5.65 \mathrm{~V}$ was achieved with a transition width of about $0.2 \mathrm{~V}$. The photographs inserted in Fig. 2(e) represent the colors of the display at input voltages of less than $5.45 \mathrm{~V}$ (white color) and more than $5.65 \mathrm{~V}$ (black color). The display became uniformly black at more than $5.65 \mathrm{~V}$, which is the value defined below as $V_{a}$.

The potentiometric response of the electrode modified with $\mathrm{LOx} / \mathrm{PB}$ for various L-lactate concentrations was evaluated using the setup in Fig. 3(a). The extended-gate and $\mathrm{Ag} / \mathrm{AgCl}$ reference electrodes were immersed in the DPBS solution. The difference in electrical potential between these electrodes was monitored using a digital voltmeter. This difference increased linearly with time after the injection of L-lactate [representative data are shown in the graph inserted within Fig. 4(b)] because the electrode connected to a high-input impedance digital voltmeter in series induces the irreversible oxidation of $\mathrm{PB} .{ }^{(28)}$ Figure $3(\mathrm{~b})$ shows the change in electrical potential difference $\Delta V_{L A C}$ as a function of L-lactate concentration. The y-axis represents $\Delta V_{L A C}$ obtained over $5 \mathrm{~min}$ after injecting L-lactate. The electrode exhibited a typical 


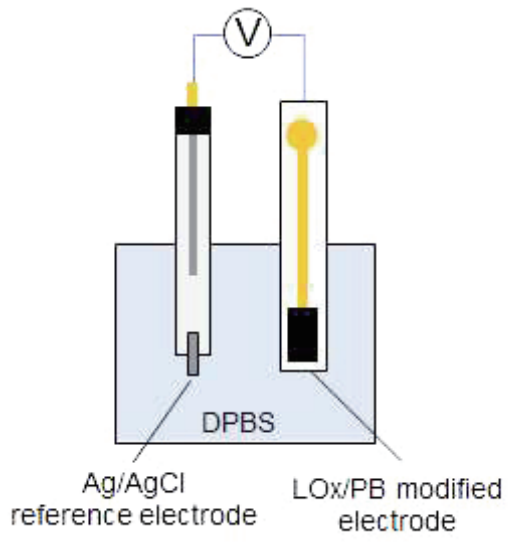

(a)

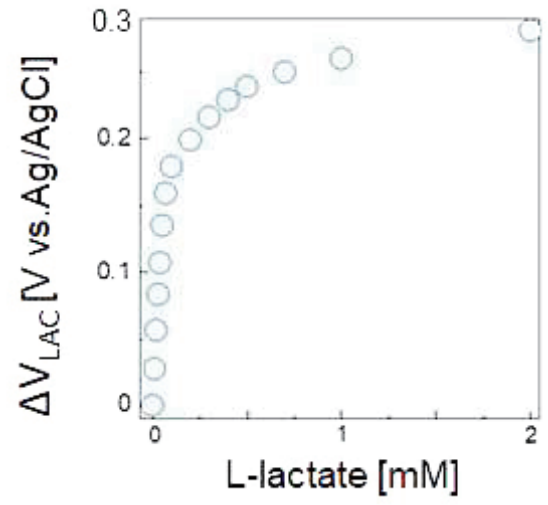

(b)

Fig. 3. (Color online) (a) Setup for the potentiometric measurement as in the text using the LOx/PB-modified electrode. (b) Changes in potential difference between the LOx/PB-modified electrode and the $\mathrm{Ag} / \mathrm{AgCl}$ reference electrode for various L-lactate concentrations in DPBS.

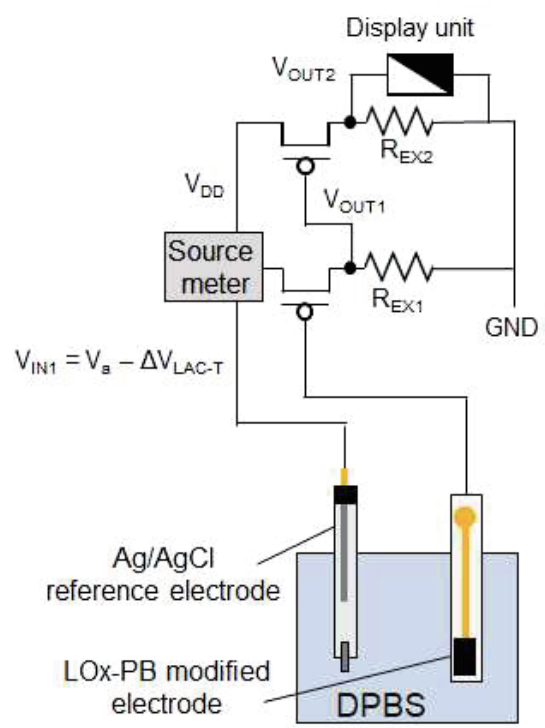

(a)

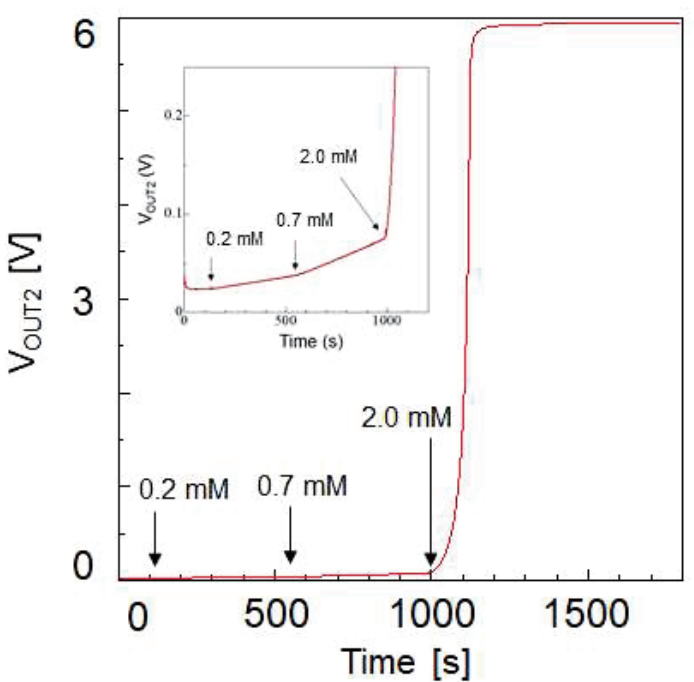

(b)

Fig. 4. (Color online) (a) Setup for the L-lactate sensor using the extended-gate-type L-lactate sensor based on an organic inverter with the E Ink display. (b) Time dependence of the output voltage $V_{\text {OUT2 }}$ of the device vs L-lactate concentration. The concentration limit for L-lactate was $2.0 \mathrm{mM}$. The arrows represent the times when a concentrated L-lactate solution was injected (also indicated were final L-lactate concentrations).

enzymatic response to the L-lactate concentration. From this curve, $\Delta V_{L A C-T}$ was calculated as $0.2,0.25$, and $0.3 \mathrm{~V}$ when the L-lactate concentration limit was defined as $0.2,0.7$, and $2.0 \mathrm{mM}$, respectively. However, the reproducibility of potentiometric response among different LOx/ PB electrodes was relatively low in the present stage owing to the irreversible redox cycling of PB as described above. Therefore, we used the same LOx/PB electrode of Fig. 3(b) for the following Fig. 4(b) experiments to demonstrate the basic operational principle of the present sensor device. 
Figure 4 shows the setup for the L-lactate sensor. The electrical characteristics of the inverter circuit connected to the electrophoretic display were evaluated immediately before use, as shown in Fig. 2(e). The extended-gate and reference electrodes were immersed in the DPBS solution, and their terminal ends were connected to the gate and source electrodes of the first stage of the inverter, respectively. $V_{I N}$ and $V_{D D}$ were controlled by using a commercially available two-channel source meter. $V_{I N 1}$ was defined as follows: $V_{I N 1}=$ $V_{a}(5.65 \mathrm{~V})-\Delta V_{L A C-T}$. To set the L-lactate concentration limit to $2.0 \mathrm{mM}, V_{I N}$ was calculated as follows: $V_{I N 1}=5.65 \mathrm{~V}-0.3 \mathrm{~V}$ [ $\Delta V_{L A C-T}$ for $2.0 \mathrm{mM} \mathrm{L}$-lactate calculated from Fig. 3(b)] or $5.35 \mathrm{~V}$. Figure 4(b) shows the time dependence of $V_{\text {OUT2 }}$ after successive injections of a concentrated L-lactate solution at 5 min intervals. $V_{O U T 2}$ gradually increased after the injection of 0.2 and $0.7 \mathrm{mM}$ L-lactate with slopes depending on the L-lactate concentration, as shown in the inset in Fig. 4(b), while $V_{\text {OUT2 }}$ attained $5 \mathrm{~min}$ after the injection of L-lactate was much smaller than the concentration-limit voltage $V_{a}$. When the L-lactate concentration was increased to $2.0 \mathrm{mM}$, the sensor exhibited a steep increase in $V_{\text {OUT2 }}$ to $6 \mathrm{~V}$, followed by a change in display color. The L-lactate concentration limit could be tuned to 0.2 and $0.7 \mathrm{mM}$ when $V_{I N 1}$ was set to $5.45 \mathrm{~V}$ $\left(V_{a}-0.2 \mathrm{~V}\right)$ and $5.4 \mathrm{~V}\left(V_{a}-0.25 \mathrm{~V}\right)$, respectively (Fig. A2). To our knowledge, this is the first report on a tunable limit-detecting biosensor using an organic inverter circuit.

The detectable minimum L-lactate overlimit concentration depends on the transition width of the organic inverter circuit and the potentiometric response of the LOx/PB electrode as described above. As the transition width of the organic inverter circuit used in Fig. 4 was about $0.2 \mathrm{~V}$, the detectable minimum L-lactate overlimit concentration was $0.2 \mathrm{mM}$ roughly estimated from the potentiometric response in Fig. 3(b). A narrower transition width can be achieved by increasing the number of inverter stages. However, as the reproducibility of the electrical characteristics of not only a LOx/PB modified electrode but also an organic inverter circuit is relatively low, the minimum detectable L-lactate overlimit concentration could not be determined quantitatively at the present stage. The reproducible fabrication of the "printed" organic inverter-circuit-based biosensor with the same electrical characteristics is a challenging issue to be solved in the printed electronics field. We are now developing an advanced printed organic inverter-circuit-based enzyme sensor that exhibits a reproducible and quantitative response for future applications.

\section{Conclusions}

In this study, we developed a printed organic inverter-circuit-based enzyme sensor with a tunable detection limit. Steep amplification characteristics of the inverter circuit at the limit voltage with a small transition width enabled the distinct detection of an overlimit signal as a color change in an E Ink electrophoretic display. However, the potentiometric response for the LOx/PB-modified electrode with an irreversible redox reaction of PB makes monitoring of the analyte overlimit concentration more challenging. In our previous study, we developed another type of extended-gate-type organic transistor-based enzyme sensor that exhibits a stepwise, stable, and reversible response to the analyte. ${ }^{(28)}$ The enzyme/PB-modified extended-gate and $\mathrm{Ag} / \mathrm{AgCl}$ reference electrodes were short-circuited via an external resistor, allowing a reversible 
redox reaction of PB. We are now developing an organic inverter-circuit-based enzyme sensor that exhibits a reversible and quantitative response for wearable chemical devices using excreted bodily fluids such as tears, urine, saliva, and sweat.

\section{Acknowledgments}

This work was partly supported by the Center of Innovation Program (COI) sponsored by the Japan Science and Technology Agency, JST, Leading Initiative for Excellent Young Researchers (LEADER), and a Grant-in-Aid for Scientific Research C (18K04910) from the Ministry of Education, Culture, Sports, Science and Technology, Japan. The semiconductor material DTBDT-C6 was provided by Tosoh Corporation.

\section{References}

1 A. Tricoli, N. Nasiri, and S. De: Adv. Funct. Mater. 27 (2018) 1605271. https://doi.org/10.1002/adfm.201605271

2 J. Kim, R. Kumar, A. J. Bandodkar, and J. Wang: Adv. Electron. Mater. 3 (2017) 1600260. https://doi. org/10.1002/aelm.201600260

3 K. Mitsubayashi and T. Arakawa: Electroanalysis 28 (2016) 1170. https://doi.org/10.1002/elan.201600083

4 S. Wang, T. Chinnasamy, M. A. Lifson, F. Inci, and U. Dermirci: Trends Biotechnol. 34 (2016) 909. https://doi. org/10.1016/j.tibtech.2016.05.009

5 J. Choi, T. W. Seong, M. Jeun, and K. H. Lee: Adv. Healthcare Mater. 6 (2017) 1700796. https://doi.org/10.1002/ adhm. 201700796

6 I. Hwang, H. N. Kim, M. Seong, S. H. Lee, M. Kang, H. Yi, W. G. Bae, M. K. Kwak, and H. E. Jeong: Adv. Healthcare Mater. 7 (2018) e1800275. https://doi.org/10.1002/adhm.201800275

7 M. Driller, N. Borges, and D. Plewa: Sports Eng. 19 (2016) 229. https://doi.org/10.1007/s12283-016-0198-6

8 P. Farzam, Z. Starweather, and M. A. Franceschini: Physiol. Rep. 6 (2018) e13664. https://doi.org/10.14814/ phy2.13664

9 C. H. Tsai, Y. J. Hong, J. H. Huang, P. Chang, and T. R. Yew: J. Microb. Biochem. Technol. 8 (2016) 5. https:// doi.org/10.4172/1948-5948.1000314

10 A. Zloczewska, A. Celebanska, K. Szot, D. Tomaszewska, M. Opallo, and M. Jönsson-Niedziolka: Biosens. Bioelectron. 54 (2014) 455. https://doi.org/10.1016/j.bios.2013.11.033

11 M. A. Pellitero, A. Guimerà, M. Kitsara, R. Villa, C. Rubio, B. Lakard, M. L. Doche, J. Y. Hihnc, and F. Javier del Campo: Chem. Sci. 8 (2017) 1995. https://doi.org/10.1039/C6SC04469G

12 H. Park, D. S. Kim, S. Y. Hong, C. Kim, J. Y. Yun, S. Y. Oh, S. W. Jin, Y. R. Jeong, G. T. Kim, and J. S. Ha: Nanoscale 9 (2017) 7631. https://doi.org/10.1039/c7nr02147j.

13 M. A. Pellitero, A. Guimerà, R. Villa, and F. Javier del Campo: J. Phys. Chem. C 122 (2018) 2596. https://doi. org/10.1021/acs.jpcc.7b11906

14 C. Wang, D. Hwang, Z. Yu, K. Takei, J. Park, T. Chen, B. Ma, and A. Javey: Nat. Mater. 12 (2013) 899. https:// doi.org/10.1038/nmat3711

15 C. Yu, Y. Li, X. Zhang, X. Huang, V. Malyarchuk, S. Wang, Y. Shi, L. Gao, Y. Su, Y. Zhang, H. Xu, R. T. Hanlon, Y. Huang, and J. A. Rogers: Proc. Natl. Acad. Sci. USA 111 (2014) 12998. https://doi.org/10.1073/pnas.1410494111

16 T. B. Singh, P. Senkarabacak, and N. S. Sariciftci: Appl. Phys. Lett. 89 (2006) 033512. https://doi. org/10.1063/1.2235947

17 T. Sekitani, U. Zschieschang, H. Klauk, and T. Someya: Nat. Mater. 9 (2010) 1015. https://doi.org/10.1038/ nmat2896

18 P. Chen, Y. Fu, R. Aminirad, C. Wang, J. Zhang, K. Wang, K. Galatsis, and C. Zhou: Nano Lett. 11 (2011) 5301. https://doi.org/10.1021/n1202765b

19 K. Besar, J. Dailey, X. Zhao, and H. Katz: J. Mater. Chem. C 5 (2017) 6506. https://doi.org/10.1039/ C7TC01377A

20 R. Shiwaku, Y. Takeda, T. Fukuda, K. Fukuda, H. Matsui, D. Kumaki, and S. Tokito: Sci. Rep. 6 (2016) 34723. https://doi.org/ 10.1038/srep34723

21 R. Shiwaku, H. Matsui, K. Hayasaka, Y. Takeda, T. Fukuda, D. Kumaki, and S. Tokito: Adv. Electron. Mater. 3 (2017) 1600557. https://doi.org/10.1002/aelm.201600557

22 W. Honda, S. Harada, T. Arie, S. Akita, and K. Takei: Adv. Funct. Mater. 24 (2014) 3299. https://doi. org/10.1002/adfm.201303874 
23 J. Li, F. Rossignolb, and J. Macdonald: Lab Chip 15 (2015) 2538. https://doi.org/10.1039/C5LC00235D

24 M. Bariya, Z. Shahpar, H. Park, J. Sun, Y. Jung, W. Gao, H. Y. Y. Nyein, T. S. Liaw, L. C.ai, Q. P. Ngo, M. Chao, Y. Zhao, Mark. Hettick, G. Cho, and A. Javey: ACS Nano 12 (2018) 6978. https://doi.org/10.1021/ acsnano.8b02505

25 R. Shiwaku, H. Matsui, K. Nagamine, M. Uematsu, T. Mano, Y. Maruyama, A. Nomura, K. Tsuchiya, K. Hayasaka, Y. Takeda, T. Fukuda, D. Kumaki, and S. Tokito: Sci. Rep. 8 (2018) 6368. https://doi.org/10.1038/ s41598-018-24744-x

26 R. Shiwaku, H. Matsui, K. Nagamine, M. Uematsu, T. Mano, Y. Maruyama, A. Nomura, K. Tsuchiya, K. Hayasaka, Y. Takeda, T. Fukuda, D. Kumaki, and S. Tokito: Sci. Rep. 8 (2018) 3922. https://doi.org/10.1038/ s41598-018-22265-1

27 S. Wang, J. Xu, W. Wang, G. J. N. Wang, R. Rastak, F. M. Lopez, J. W. Chung, S. Niu, V. R. Feig, J. Ropez, T. Lei, S. K. Kwon, Y. Kim, A. M. Foudeh, A. Ehrlich, A. Gasperini, Y. Yun, B. Murmann, J. B. H. Tok, and Z. Bao: Nature 555 (2018) 83. https://doi.org/10.1038/nature25494

28 T. Mano, K. Nagamine, Y. Ichimura, R. Shiwaku, H. Furusawa, H. Matsui, D. Kumaki, and S. Tokito: to be published in ChemElectroChem. https://doi.org/10.1002/celc.201801129

29 A. J. Bandodkar, W. Jia, C. Yardimci, X. Wang, J. Ramirez, and J. Wang: Anal. Chem. 87 (2015) 394. https:// doi.org/10.1021/ac504300n

30 J. Kim, J. R. Sempionatto, S. Imani, M. C. Hartel, A. Barfidokht, G. Tang, A. S. Campbell, P. P. Mercier, and J. Wang: Adv. Sci. 5 (2018) 1800880. https://doi.org/10.1002/advs.201800880

\section{Appendix}

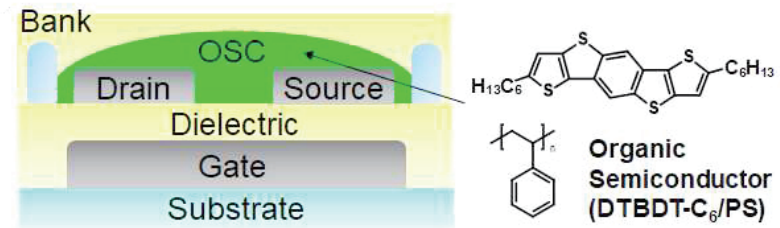

(a)

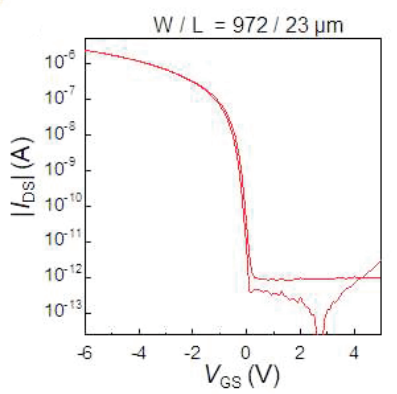

(b)

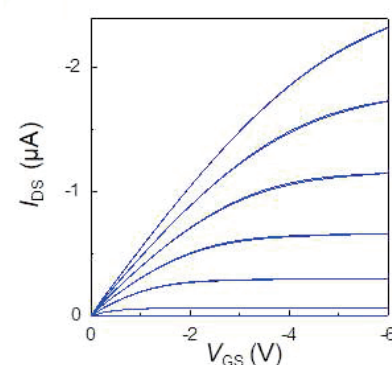

(c)

Fig. A1. (Color online) (a) Structure of the fabricated organic transistor. (b) Transfer and (c) output curves of a single organic transistor.

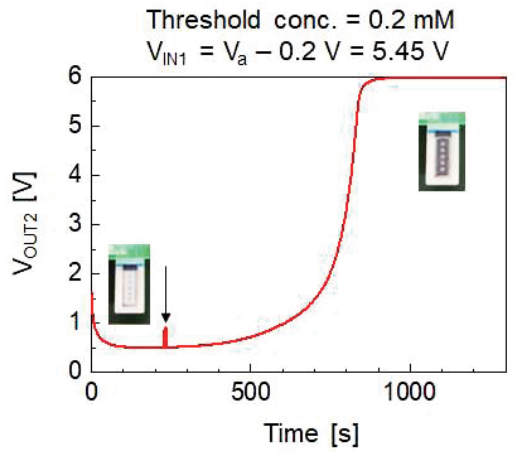

(a)

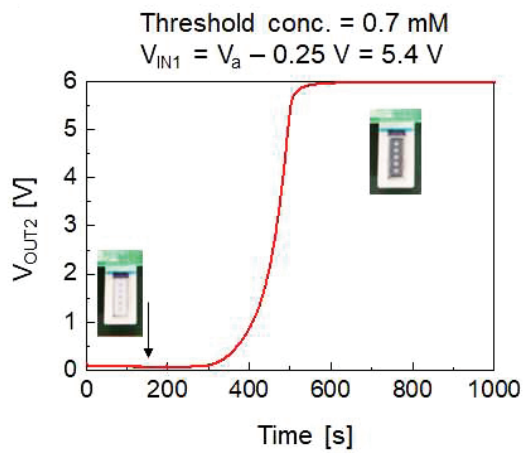

(b)

Fig. A2. (Color online) Time dependence of the output voltage VOUT2 for a device with L-lactate concentration limits of (a) 0.2 and (b) $0.7 \mathrm{mM}$. 